\section{Continuous Finite-Time Stabilization of the Translational and Rotational Double Integrators}

Sanjay P. Bhat and Dennis S. Bernstein

\begin{abstract}
A class of bounded continuous time-invariant finite-time stabilizing feedback laws is given for the double integrator. Lyapunov theory is used to prove finite-time convergence. For the rotational double integrator, these controllers are modified to obtain finite-time-stabilizing feedbacks that avoid "unwinding."
\end{abstract}

Index Terms - Finite-time stability, non-Lipschitzian dynamics, stability, stabilization.

\section{INTRODUCTION}

Most of the available techniques for feedback stabilization lead to closed-loop systems with Lipschitzian dynamics. The convergence in such systems is at best exponential with infinite settling time. In other words, none of the solutions starting in an open neighborhood of the origin converge to the origin in finite time. In fact, finitetime convergence implies nonuniqueness of solutions (in reverse time) which is not possible in the presence of Lipschitz-continuous dynamics.

Our goal is to develop techniques for obtaining continuous finitetime-stabilizing feedback controllers. The present paper focuses on the double integrator as an illustrative example of this objective.

Since the double integrator is controllable, open-loop control strategies can be used to drive the state to the origin in finite time [1, p. 38]. One such control strategy is the minimum energy control [2], which transfers the state of the system $\ddot{x}=u$ from the initial conditions $x(0)=x_{0}, \dot{x}(0)=y_{0}$ to the origin in a given time $t_{f}$. This control strategy minimizes the control energy cost $J(u)=\int_{0}^{t} f(u(t))^{2} d t$ and is given by [2, pp. 466-475]

$$
u\left(x_{0}, y_{0}, t\right)=-\frac{2}{t_{f}^{2}}\left(3 x_{0}+2 y_{0} t_{f}\right)+\frac{6}{t_{f}^{3}}\left(2 x_{0}+y_{0} t_{f}\right) t .
$$

Similar finite-settling-time open-loop controls for linear systems are given in [3] and some of the references contained therein. Open-loop strategies, however, are generally sensitive to system uncertainties and may perform poorly in the presence of disturbances.

Finite-time-stabilizing feedback laws can be obtained through optimal synthesis. A well-known example is the bang-bang timeoptimal controller. In [4] and [5], it is shown that the minimization of certain nonquadratic cost functionals subject to a saturation constraint on the control input yields finite-time-stabilizing feedback controllers. In fact, it is shown in [5] that the time-optimal controller for the double integrator also minimizes a nonquadratic cost functional. This property of the time-optimal controller is used in [6] to derive a finite-time-stabilizing feedback controller for uncertain scalar secondorder systems. One drawback of the controllers given in [4]-[6] is that, like the time-optimal controller, they are discontinuous. For certain initial conditions, some of the controllers in [4] and [5]

Manuscript received February 15, 1996. This work was supported in part by the Air Force Office of Scientific Research under Grant F49620-95-1-0019.

S. P. Bhat is with the Department of Aerospace Engineering, Indian Institute of Technology, Powai, Mumbai 400076 India.

D. S. Bernstein is with the Department of Aerospace Engineering, University of Michigan, Ann Arbor, MI 48109-2118 USA (e-mail: dsbaero@engin.umich.edu).

Publisher Item Identifier S 0018-9286(98)03606-X. even yield control functions with a countably infinite number of discontinuities. In practical implementations, discontinuous feedback controllers can lead to chattering behavior due to plant uncertainties or measurement imperfections. Such controllers may also excite unmodeled high-frequency dynamics when used, for instance, to control lightly damped structures [7].

The design of continuous time-invariant finite-time-stabilizing feedback controllers presents a challenge because such controllers necessarily involve non-Lipschitzian closed-loop dynamics. Consequently, relatively little attention has been paid to such controllers even though there are indications that finite-time-stable closed-loop systems might have better robustness and disturbance rejection properties [8]. While a constructive method for obtaining finitetime-stabilizing controllers for the double integrator is given in [9], the applicability of this method is limited by the fact that the construction involves finding closed-form analytical solutions to algebraic equations. A class of finite-time-stabilizing controllers for linear systems appears in [10]. This class of controllers is limited to systems having as many control inputs as state variables and hence cannot be applied to the double integrator. A family of continuous time-invariant finite-time-stabilizing controllers for the double integrator is proposed in [11]. These controllers are unbounded, while the proofs use constructions special to the case of the double integrator.

In Section III of this paper we give a family of continuous time-invariant finite-time-stabilizing feedback laws for the double integrator. In contrast to [11], we demonstrate finite-time stabilization directly by constructing a suitable Lyapunov function. In Section IV we give a class of globally bounded feedback laws for the finite-time stabilization of the double integrator. The results are based on the Lyapunov theory for finite-time differential equations developed in [12]. This theory is briefly presented in Section II for completeness.

As an extension of these results, we consider a rigid body rotating under the action of a control torque about a fixed axis. Such a rigid body has equations of motion that resemble those of a double integrator. However, since states that differ by integral multiples of $2 \pi$ in angular position correspond to the same physical configuration of the body, the state space for this rotational double integrator is the two-dimensional cylinder $S^{1} \times \mathbb{R}$ rather than $\mathbb{R}^{2}$ [13, p. 419]. Stabilizing control laws developed for the double integrator on $\mathbb{R}^{2}$ (the translational double integrator) when applied to the rotational double integrator lead to the "unwinding" phenomenon in which the body may rotate numerous times before coming to rest, even if the initial configuration is the same as the final desired one. In spacecraft applications, such unwinding can lead to the inefficient use of momentum-management devices and fuel. Hence the design of controllers that finite-time stabilize the rotational double integrator without causing unwinding is of special interest. In Section V, we present such a class of finite-time-stabilizing feedback controllers for the rotational double integrator.

The results of Sections III-V also appear in [14], which is a preliminary version of the present paper.

\section{Finite-Time StabiLITy}

Consider the system

$$
\dot{y}(t)=f(y(t))
$$

where $f: \mathcal{D} \rightarrow \mathbb{R}^{n}$ is continuous on an open neighborhood $\mathcal{D}$ of the origin and $f(0)=0$. A continuously differentiable function 
$y: I \rightarrow \mathcal{D}$ is said to be a solution of (1) on the interval $I \subseteq \mathbb{R}$ if $y$ satisfies (1) for all $t \in I$. The continuity of $f$ implies that for every $x \in \mathcal{D}$, there exist $\tau_{0}<0<\tau_{1}$ and a solution $y(\cdot)$ of (1) defined on $\left(\tau_{0}, \tau_{1}\right)$ such that $y(0)=x[15$, pp. 10-11]. We will assume that $(1)$ possesses unique solutions in forward time for all initial conditions, except possibly the origin; that is, for every $x \in \mathcal{D} \backslash\{0\}$ there exist $\tau_{2}>0$ and a unique solution $y(\cdot)$ of (1) defined on $\left[0, \tau_{2}\right)$ and satisfying $y(0)=x$. In this case we denote the unique solution $y(\cdot)$ of (1) satisfying $y(0)=x \in \mathcal{D} \backslash\{0\}$ by $\rho_{(\cdot)}(x)$, that is, $\rho_{t}(x)=y(t)$.

Remark 1: The uniqueness assumption is of more than just academic interest. Uniqueness in forward time and the continuity of $f$ ensure that solutions are continuous functions of initial conditions even when $f$ is not Lipschitz continuous (see [15, Th. 2.1, p. 94]).

Definition: The origin is said to be a finite-time-stable equilibrium of (1) if there exists an open neighborhood $\mathcal{N} \subseteq \mathcal{D}$ of the origin and a function $T: \mathcal{N} \backslash\{0\} \rightarrow(0, \infty)$, called the settling time, such that the following statements hold.

1) Finite-time convergence: For every $x \in \mathcal{N} \backslash\{0\}, \rho_{t}(x)$ is defined for $t \in[0, T(x)), \rho_{t}(x) \in \mathcal{N} \backslash\{0\}$, for $t \in[0, T(x))$, and $\lim _{t \rightarrow T(x)} \rho_{t}(x)=0$.

2) Lyapunov stability: For every open set $\mathcal{U}_{\varepsilon}$ such that $0 \in \mathcal{U}_{\varepsilon} \subseteq$ $\mathcal{N}$, there exists an open set $\mathcal{U}_{\delta}$ such that $0 \in \mathcal{U}_{\delta} \subseteq \mathcal{N}$ and such that for every $x \in \mathcal{U}_{\delta} \backslash\{0\}, \rho_{t}(x) \in \mathcal{U}_{\varepsilon}$ for $t \in[0, T(x))$.

The origin is said to be a globally finite-time-stable equilibrium if it is a finite-time-stable equilibrium and $\mathcal{D}=\mathcal{N}=\mathbb{R}^{n}$.

Versions of the following result have appeared in [6], [11], and [12].

Theorem 1: Suppose there exists a continuously differentiable function $V: \mathcal{D} \rightarrow \mathbb{R}$, real numbers $k>0$ and $\alpha \in(0,1)$, and a neighborhood $\mathcal{U} \subset \mathcal{D}$ of the origin such that $V$ is positive definite on $\mathcal{U}$ and $\dot{V}+k V^{\alpha}$ is negative semidefinite on $\mathcal{U}$, where $\dot{V}(x)=$ $\frac{\partial V}{\partial x}(x) f(x)$. Then the origin is a finite-time-stable equilibrium of (1). Moreover, if $T$ is the settling time, then $T(x) \leq \frac{1}{k(1-\alpha)} V(x)^{1-\alpha}$ for all $x$ in some open neighborhood of the origin.

\section{Continuous Finite-Time-Stabilizing Controllers}

In this section, we present a class of continuous time-invariant feedback controllers that globally finite-time stabilize the double integrator. By a finite-time-stabilizing feedback law, we mean a feedback controller that renders the origin of the closed-loop system a finite-time-stable equilibrium as defined in the previous section. Thus we seek a continuous feedback law

$$
u=\psi(x, y)
$$

that finite-time stabilizes the double integrator

$$
\dot{x}=y, \quad \dot{y}=u .
$$

Proposition 1: The origin of (3) is a globally finite-time-stable equilibrium under the feedback control law (2) with

$$
\psi(x, y)=-\operatorname{sign}(y)|y|^{\alpha}-\operatorname{sign}\left(\phi_{\alpha}(x, y)\right)\left|\phi_{\alpha}(x, y)\right|^{\frac{\alpha}{2-\alpha}}
$$

for every $\alpha \in(0,1)$, where $\phi_{\alpha}(x, y) \triangleq x+\frac{1}{2-\alpha} \operatorname{sign}(y)|y|^{2-\alpha}$.

Proof: Fix $\alpha \in(0,1)$. For convenience denote $\phi_{\alpha}(x, y)$ by $\phi_{\alpha}$ and consider the continuously differentiable Lyapunov function candidate

$$
V(x, y)=\frac{2-\alpha}{3-\alpha}\left|\phi_{\alpha}\right|^{\frac{3-\alpha}{2-\alpha}}+s y \phi_{\alpha}+\frac{r}{3-\alpha}|y|^{3-\alpha}
$$

where $r$ and $s$ are positive numbers.

Along the closed-loop trajectories, we have

$$
\begin{aligned}
\dot{V}(x, y)= & -r y^{2}-s\left|\phi_{\alpha}\right|^{\frac{2}{2-\alpha}}-|y|^{1-\alpha}\left|\phi_{\alpha}\right|^{\frac{1+\alpha}{2-\alpha}} \\
& -s \phi_{\alpha} \operatorname{sign}(y)|y|^{\alpha}-(r+s) \operatorname{sign}\left(y \phi_{\alpha}\right)|y|^{2-\alpha}\left|\phi_{\alpha}\right|^{\frac{\alpha}{2-\alpha}}
\end{aligned}
$$

which is continuous everywhere since $\alpha \in(0,1)$. It is easy to verify that, for $k>0$ and $(x, y) \in \mathbb{R}^{2}$

$$
\begin{aligned}
& V\left(k^{2-\alpha} x, k y\right)=k^{3-\alpha} V(x, y) \\
& \dot{V}\left(k^{2-\alpha} x, k y\right)=k^{2} \dot{V}(x, y) .
\end{aligned}
$$

It can easily be shown that, for $r>1$ and $s<1$, both $V$ and $-\dot{V}$ take positive values on the set $\mathcal{O}=\{(x, y)$ : $\left.\max _{(x, y) \neq(0,0)}\left\{\left|\phi_{\alpha}\right|^{\frac{1}{2-\alpha}},|y|\right\}=1\right\}$ which is a closed curve encircling the origin. Since for every $(x, y) \in \mathbb{R}^{2} \backslash\{(0,0)\}$ there exists $k>0$ such that $\left(k^{2-\alpha} x, k y\right) \in \mathcal{O}$, the homogeneity properties (6) and (7) imply that $V$ is positive definite and $\dot{V}$ is negative definite.

It also follows from (6) that $V$ is radially unbounded so that the set $\mathcal{V}=\{(x, y): V(x, y)=1\}$ is compact. Since $\dot{V}$ is continuous, $\dot{V}$ achieves its maximum on the compact set $\mathcal{V}$. Define $c=-\max _{(x, y) \in \mathcal{V}} \dot{V}(x, y)$ and note that $c>0$ since $-\dot{V}$ is positive definite and $(0,0) \notin \mathcal{V}$. The homogeneity properties (6) and (7) can now be used to show that $\dot{V}(x, y) \leq-c\{V(x, y)\}^{\frac{2}{3-\alpha}}$ for all $(x, y) \in \mathbb{R}^{2}$; see, for instance, [6] and [14]. Since $\alpha \in(0,1)$ is equivalent to $\frac{2}{3-\alpha} \in(0,1)$, finite-time stability of the origin follows from [11, Th. 1 in Section I or Proposition 1]. Global stability holds since $V$ is radially unbounded and $\dot{V}$ is negative definite.

Remark 2: The closed-loop vector field obtained by using the feedback control law (4) is locally Lipschitz everywhere except the $x$-axis, denoted $\mathcal{X}$, and the zero-level set $\mathcal{S}=\left\{(x, y): \phi_{\alpha}(x, y)=\right.$ $0\}$ of the function $\phi_{\alpha}$. Since the closed-loop vector field $f_{\alpha}$ is transversal to $\mathcal{X}$ at every point in $\mathcal{X} \backslash\{(0,0)\}$, it follows from [16, Proposition 2.2] or [17, Lemma 2, p. 107] that every initial condition in $\mathcal{X} \backslash\{(0,0)\}$ has a unique solution in forward time. The set $\mathcal{S}$ is a positively invariant set for the closed-loop dynamics, that is, every solution $(x(\cdot), y(\cdot))$ of the closed-loop system satisfies $(x(t), y(t)) \in$ $\mathcal{S}$ for $t \geq 0$ if $(x(0), y(0)) \in \mathcal{S}$. This follows by noting that along the closed-loop solutions $\phi_{\alpha} \dot{\phi}_{\alpha}=-|y|^{1-\alpha}\left|\phi_{\alpha}(x, y)\right|^{\frac{2}{2-\alpha}} \leq 0$ for every $(x, y) \in \mathbb{R}^{2}$. Moreover, on $\mathcal{S}$, the closed-loop system is given by

$$
\begin{aligned}
& \dot{x}=-\operatorname{sign}(x)[(2-\alpha)|x|]^{\frac{1}{2-\alpha}} \\
& \dot{y}=-\operatorname{sign}(y)|y|^{\alpha} .
\end{aligned}
$$

System (8) is locally Lipschitz everywhere except the origin and therefore possesses unique solutions in forward time for initial conditions in $\mathcal{S} \backslash\{(0,0)\}$. Thus the closed-loop system satisfies the uniqueness assumption made in Section I.

Example 1: Fig. 1 shows the phase portrait of the double integrator under the feedback law

$$
\psi(x, y)=-y^{\frac{1}{3}}-\left(x+\frac{3}{5} y^{\frac{5}{3}}\right)^{\frac{1}{5}}
$$

which is obtained from (4) by using $\alpha=\frac{1}{3}$. An interesting feature of the closed-loop system is that all trajectories converge to the set $\mathcal{S}=\left\{(x, y): x+\frac{3}{5} y^{\frac{5}{3}}=0\right\}$ in finite time. The set $\mathcal{S}$ is positively invariant and represents what is called a terminal sliding mode in [8]. The term $-y^{\frac{1}{3}}$ in (9) renders $\mathcal{S}$ positively invariant while the remaining term $-\left(x+\frac{3}{5} y^{\frac{5}{3}}\right)^{\frac{1}{5}}$ in (9) drives states to $\mathcal{S}$ in finite-time. The controller (4) is thus an example of sliding mode control without using discontinuous or high-gain feedback.

\section{Bounded Continuous Finite-Time Controllers}

The finite-time-stabilizing controllers developed in the previous section were unbounded. In this section, we modify the class of controllers introduced in the last section to obtain a class of bounded continuous time-invariant feedback controllers that globally finitetime stabilize the double integrator. The modification consists of saturating various terms in the controller (4). 


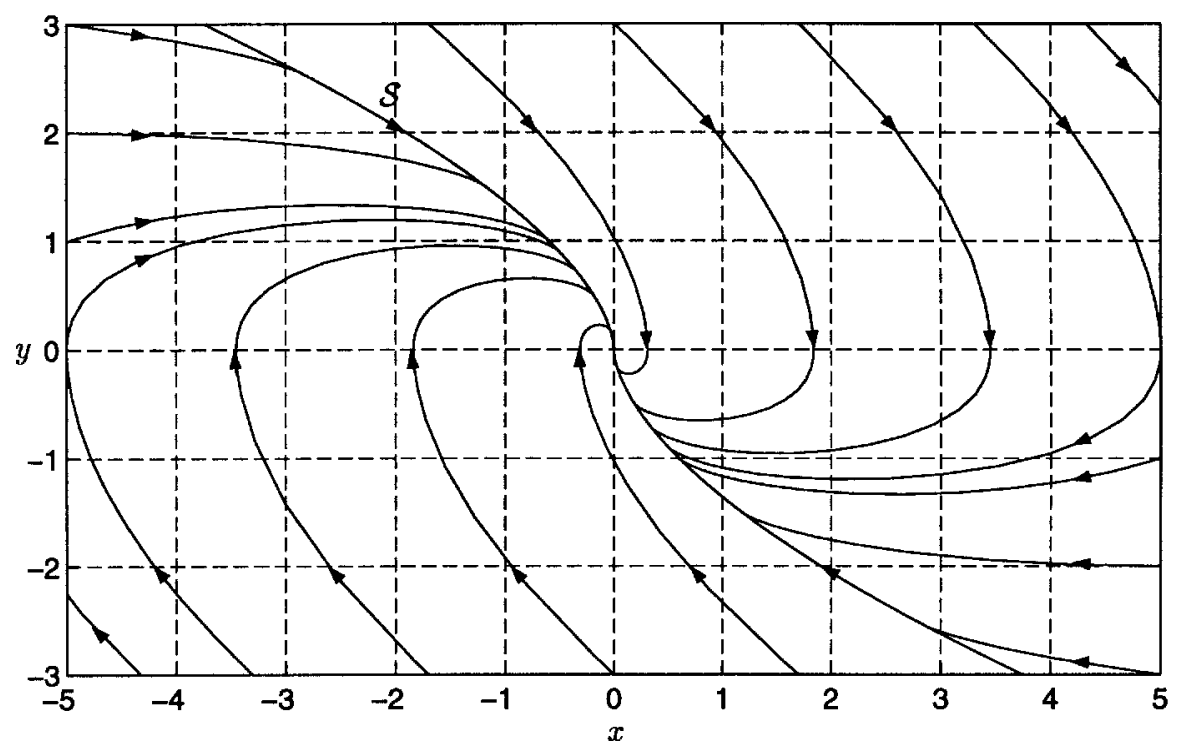

Fig. 1. Double integrator with the controller (4).

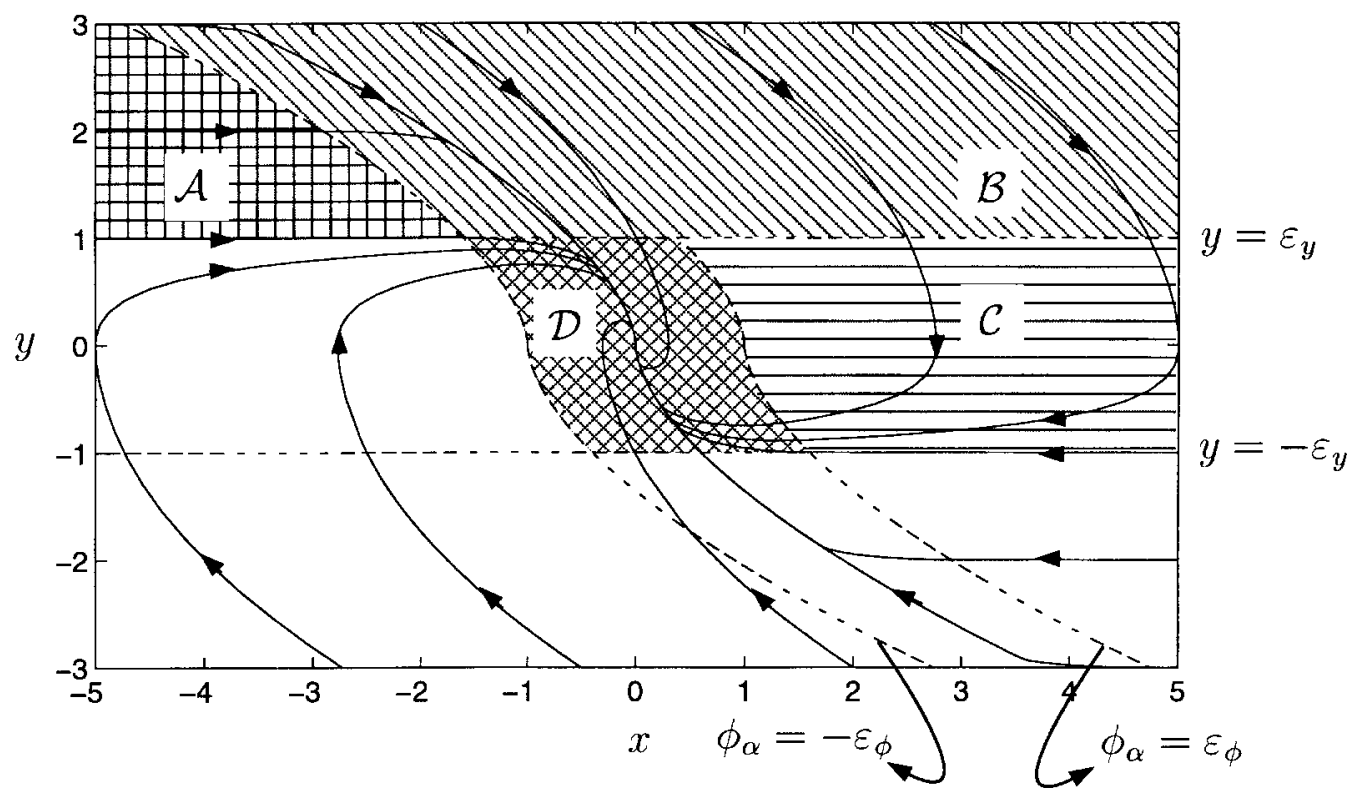

Fig. 2. Double integrator with the controller (10).

Given a positive number $\varepsilon$, define

$$
\begin{aligned}
\operatorname{sat}_{\varepsilon}(y) & =y, \quad|y|<\varepsilon \\
& =\varepsilon \operatorname{sign}(y), \quad|y| \geq \varepsilon .
\end{aligned}
$$

Note that $\left|\operatorname{sat}_{\varepsilon}(y)\right| \leq \varepsilon$ for all $y \in \mathbb{R}$.

Proposition 2: The origin of (3) is a globally finite-time-stable equilibrium under the bounded feedback control law $u=\psi_{\text {sat }}(x, y)$ with

$$
\begin{aligned}
\psi_{\text {sat }}(x, y)= & -\operatorname{sat}_{\varepsilon}\left\{\operatorname{sign}(y)|y|^{\alpha}\right\} \\
& -\operatorname{sat}_{\varepsilon}\left\{\operatorname{sign}\left(\phi_{\alpha}(x, y)\right)\left|\phi_{\alpha}(x, y)\right|^{\frac{\alpha}{2-\alpha}}\right\}
\end{aligned}
$$

for every $\alpha \in(0,1)$ and $\varepsilon>0$, where $\phi_{\alpha}(x, y) \triangleq x+$ $\frac{1}{2-\alpha} \operatorname{sign}(y)|y|^{2-\alpha}$.

Proof: Let $\alpha \in(0,1)$ and $\varepsilon>0$. The boundedness of $\psi_{\text {sat }}$ follows from the triangle inequality. To prove global finite-time stability, we define the sets

$$
\begin{aligned}
\mathcal{A} & =\left\{(x, y): y \geq \varepsilon_{y}, \phi_{\alpha}(x, y)<-\varepsilon_{\phi}\right\} \\
\mathcal{B} & =\left\{(x, y): y>\varepsilon_{y}, \phi_{\alpha}(x, y) \geq-\varepsilon_{\phi}\right\} \\
\mathcal{C} & =\left\{(x, y):|y| \leq \varepsilon_{y}, \phi_{\alpha}(x, y)>\varepsilon_{\phi}\right\} \\
\mathcal{D} & =\left\{(x, y):|y| \leq \varepsilon_{y},\left|\phi_{\alpha}(x, y)\right| \leq \varepsilon_{\phi}\right\}
\end{aligned}
$$

where $\varepsilon_{y}=\varepsilon^{\frac{1}{\alpha}}$ and $\varepsilon_{\phi}=\varepsilon^{\frac{2-\alpha}{\alpha}}$. Fig. 2 depicts these sets for clarity. For convenience, denote $\phi_{\alpha}(x, y)$ by $\phi_{\alpha}$ and $\frac{\partial \phi_{\alpha}}{\partial x}(x, y) \dot{x}+$ $\frac{\partial \phi_{\alpha}}{\partial y}(x, y) \dot{y}$ by $\dot{\phi}_{\alpha}$.

The boundary of $\mathcal{D}$, bd $\mathcal{D}=\left\{(x, y) \in \mathcal{D}:|y|=\varepsilon_{y}\right\} \cup\{(x, y) \in$ $\left.\mathcal{D}:\left|\phi_{\alpha}\right|=\varepsilon_{\phi}\right\}$. It is easy to verify that on $\left\{(x, y) \in \mathcal{D}:|y|=\varepsilon_{y}\right\}$, $y \dot{y} \leq 0$ while on $\left\{(x, y) \in \mathcal{D}:\left|\phi_{\alpha}\right|=\varepsilon_{\phi}\right\}, \phi_{\alpha} \dot{\phi}_{\alpha} \leq 0$. It thus follows that closed-loop solutions cannot leave $\mathcal{D}$ in forward time, that is, $\mathcal{D}$ is positively invariant. However, on $\mathcal{D}$, (4) and (10) are identical, that is, $\psi_{\text {sat }}(x, y)=\psi(x, y)$ for all $(x, y) \in \mathcal{D}$. Hence 
it follows from Proposition 1 that the origin is a finite-time-stable equilibrium.

The proof of global stability consists of showing that all trajectories starting outside $\mathcal{D}$ enter $\mathcal{D}$ after a finite amount of time. It can be shown that after a finite amount of time (that depends on the initial condition), every trajectory starting in $\mathcal{A}(-\mathcal{A})$ enters $\mathcal{B}(-\mathcal{B})$, every trajectory starting in $\mathcal{B}(-\mathcal{B})$ enters $\mathcal{C} \cup \mathcal{D}(-\mathcal{C} \cup-\mathcal{D})$, and every trajectory starting in $\mathcal{C}(-\mathcal{C})$ enters $\mathcal{D}$, where $-\mathcal{A}=$ $\{(x, y):(-x,-y) \in \mathcal{A}\}$, etc. The proof, which appears in [14], is straightforward but tedious and is omitted in the interests of space. Since every point outside $\mathcal{D}$ lies in $\mathcal{A} \cup \mathcal{B} \cup \mathcal{C} \cup-\mathcal{A} \cup-\mathcal{B} \cup-\mathcal{C}$, it follows that the origin is globally finite-time stable.

Example 2: Fig. 2 shows the phase portrait for the double integrator under the bounded feedback control law

$$
\psi_{\text {sat }}(x, y)=-\operatorname{sat}_{1}\left(y^{\frac{1}{3}}\right)-\operatorname{sat}_{1}\left\{\left(x+\frac{3}{5} y^{\frac{5}{3}}\right)^{\frac{1}{5}}\right\}
$$

which is obtained from (10) with $\alpha=\frac{1}{3}$ and $\varepsilon=1$. In this case, as in Example 1, all trajectories converge to the set $\mathcal{S}$ defined in Example 1 above. However, in certain regions of the phase plane, $\psi_{\text {sat }}(x, y)=0$. This constant velocity "coasting" behavior of the closed-loop system is similar to that of the closed-loop system obtained by using the fuel-optimal controller for the double integrator [2, pp. 675-703].

\section{The Rotational Double Integrator}

The motion of a rigid body rotating about a fixed axis with unit moment of inertia is governed by

$$
\ddot{\theta}(t)=u(t)
$$

where $\theta$ is the angular displacement from some reference and $u$ is the control torque. Equation (12) can be rewritten in the form (3) by substituting $x=\theta$ and $y=\dot{\theta}$. However, for every $(x, y) \in \mathbb{R}^{2}$, the states $(x \pm 2 n \pi, y), n=0,1,2, \cdots$, correspond to the same physical state of the rigid body. The state space for the rotational double integrator is, therefore, the two-dimensional cylinder $S^{1} \times \mathbb{R}$ rather than $\mathbb{R}^{2}[13]$.

Some applications might require that a particular angular position, say $\theta=0$, of the rigid body be rendered finite-time stable. In terms of (3), this requirement means that each of the physically identical states $( \pm 2 n \pi, 0), n=0,1,2, \cdots$ in $\mathbb{R}^{2}$ has to be a finite-timestable equilibrium. The feedback controller (4) given in Proposition 1, however, finite-time stabilizes only the origin. This feedback law, if applied to the rotational double integrator, leads to the unwinding phenomenon. Unwinding can be understood by considering the initial condition $(4 \pi, 0)$. This initial condition coincides with the desired final angular position of the rigid body and no further control action is needed. However, the feedback controller (4) takes the state $(x, y)$ from $(4 \pi, 0)$ to $(0,0)$, causing the rigid body to rotate at least twice before coming to rest in the configuration it started in. For spacecraft applications, unwinding is highly undesirable from the point of view of vibration suppression and fuel consumption. Hence, feedback controllers developed for the translational double integrator are not always suitable for the rotational double integrator so that finite-time stabilization of the rotational double integrator deserves special consideration.

From the preceding discussion, it is clear that a feedback controller for the rotational double integrator will not lead to the unwinding phenomenon if it is periodic in $x$ with period $2 \pi$. One such feedback law can be obtained by modifying (4) to

$$
\begin{aligned}
\psi_{\operatorname{rot}}(x, y)= & -\operatorname{sign}(y)|y|^{\alpha} \\
& -\operatorname{sign}\left(\sin \left(\phi_{\alpha}(x, y)\right)\right)\left|\sin \left(\phi_{\alpha}(x, y)\right)\right|^{\frac{\alpha}{2-\alpha}}
\end{aligned}
$$

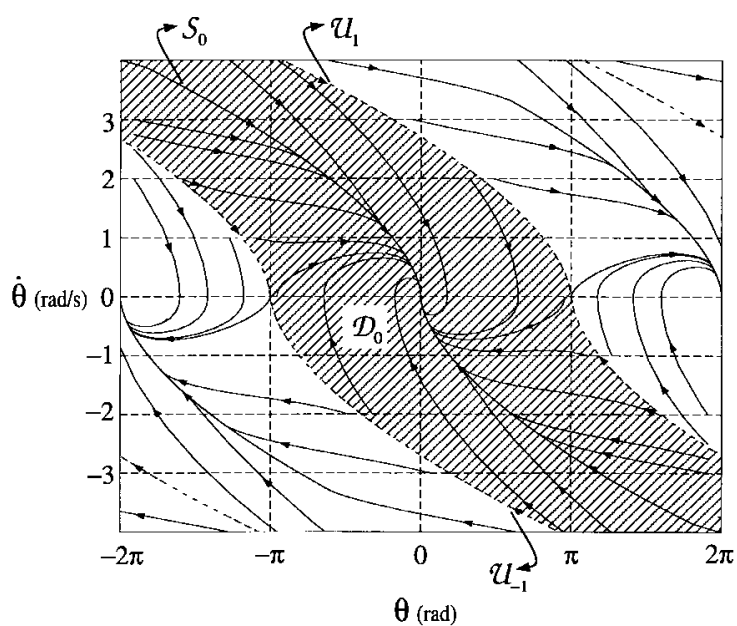

Fig. 3. Rotational double integrator with the controller (13).

where $\phi_{\alpha}(x, y)$ is as given in Proposition 1. The phase portrait for the closed-loop system (3) with the feedback $u=\psi_{\text {rot }}(x, y)$ is shown in Fig. 3 for $\alpha=\frac{1}{3}$.

The phase portrait in Fig. 3 reveals several interesting features of the closed-loop system. The closed-loop system has equilibrium points at $s_{n}=(2 n \pi, 0), u_{n}=((2 n+1) \pi, 0), \quad n=$ $\cdots,-1,0,1, \cdots$. Of these, the equilibrium points $s_{n}$ are (locally) finite-time stable in forward time, while the points $u_{n}$ are finitetime saddles, that is, for every $n$ there exist solutions that converge to $u_{n}$ in finite time in forward time and solutions that do likewise in reverse time. For a given $\alpha \in(0,1)$, the set $\mathcal{D}_{n}=\{(x, y)$ : $\left.(2 n-1) \pi<\phi_{\alpha}(x, y)<(2 n+1) \pi\right\}$ is the domain of attraction of the equilibrium point $s_{n}$. The shaded region in Fig. 3 represents a portion of $\mathcal{D}_{0}$. The sets $\mathcal{U}_{n-1}$ and $\mathcal{U}_{n}$, where $\mathcal{U}_{n}=\{(x, y)$ : $\left.\phi_{\alpha}(x, y)=(2 n+1) \pi\right\}, n=\cdots,-1,0,1, \cdots$, form the boundary of $\mathcal{D}_{n}$ and are the stable manifolds of the equilibrium points $u_{n-1}$ and $u_{n}$, respectively. All trajectories starting in the set $\mathcal{D}_{n}$ converge in finite time to the set $\mathcal{S}_{n}=\left\{(x, y): \phi_{\alpha}(x, y)=2 n \pi\right\}$ in forward time and to the set $\mathcal{U}_{n-1} \cup \mathcal{U}_{n}$ in reverse time. Moreover, the sets $\mathcal{S}_{n}$ are positively invariant, while the sets $\mathcal{U}_{n}$ are negatively invariant. The sets $\mathcal{S}_{0}, \mathcal{U}_{-1}$, and $\mathcal{U}_{1}$ are labeled in Fig. 3 for clarity.

A novel feature of the closed-loop system is the extreme nonuniqueness of solutions to initial conditions lying in any of the sets $\mathcal{U}_{n}, n=\cdots,-1,0,1, \cdots$. For any given initial condition in the set $\mathcal{U}_{n}$ and for every $\tau>0$, there exist two closed-loop solutions starting from that initial condition such that both stay in $\mathcal{U}_{n}$ until exactly time $\tau$, and then one enters the set $\mathcal{D}_{n}$ and the other enters the set $\mathcal{D}_{n+1}$. One such initial condition is the equilibrium point $u_{n}$. Every solution starting from $u_{n}$ corresponds to the rigid body resting in an unstable configuration and then spontaneously begins to move clockwise or counterclockwise. There exists a solution exhibiting every given departure time. Departure from an unstable equilibrium is a feature unique to non-Lipschitzian systems. Lipschitzian systems do not possess solutions that depart from an equilibrium.

It should be pointed out that the desired final configuration is not globally stable because of the presence of an unstable equilibrium configuration at $\theta=\pi$ corresponding to the saddle points $u_{n}, n=\cdots,-1,0,1, \cdots$. This defect, however, is shared by every continuous controller that stabilizes the rotational double integrator without causing unwinding. This is because the desired configuration corresponds to multiple equilibria in the phase plane, and thus every controller that stabilizes the desired configuration stabilizes each of these equilibria. Stability, continuous dependence on initial 
conditions, and uniqueness of solutions imply that the domains of attraction of any two distinct equilibrium points in the plane are nonempty, open and disjoint. Since $\mathbb{R}^{2}$ cannot be written as the union of a collection of disjoint open sets, it follows that there exist initial conditions in the plane that do not converge to any of the equilibria corresponding to the desired configuration. In the case of (13), these initial conditions make up the stable manifold of the unstable configuration.

The controller (13) has the advantage that the non-Lipschitzian character of the resulting closed-loop system makes the unstable configuration extremely sensitive to perturbations. This sensitivity is brought out by the fact that the closed-loop system admits solutions that depart spontaneously from the unstable configuration, even in the absence of external perturbations. Unlike in the Lipschitzian case where solutions starting sufficiently close to an unstable equilibrium stay in a given neighborhood of the equilibrium for arbitrarily long periods of time, solutions of the non-Lipschitzian closed-loop system that depart from the unstable equilibrium leave every small neighborhood of the unstable equilibrium and converge to the stable configuration in a bounded amount of time. Thus the non-Lipschitzian nature of the closed-loop system renders the state of rest at the desired configuration a globally stable equilibrium for all practical purposes.

\section{REFERENCES}

[1] W. M. Wonham, Linear Multivariable Control: A Geometric Approach, 3rd ed. New York: Springer-Verlag, 1985

[2] M. Athans and P. L. Falb, Optimal Control: An Introduction to the Theory and Its Applications. New York: McGraw-Hill, 1966.

[3] S. Choura, "Design of finite time settling regulators for linear systems," ASME J. Dynamic Syst., Measurement, and Contr., vol. 116, pp. 602-609, Dec. 1994.

[4] A. T. Fuller, "Optimization of some nonlinear control systems by means of Bellman's equation and dimensional analysis," Int. J. Contr., vol. 3 , no. 4, pp. 359-394, 1966.

[5] E. P. Ryan, "Singular optimal controls for second-order saturating systems," Int. J. Contr., vol. 30, no. 4, pp. 549-564, 1979.

[6] __ "Finite-time stabilization of uncertain nonlinear planar systems," Dynamics and Contr., vol. 1, pp. 83-94, 1991.

[7] H. Baruh and S. S. K. Tadikonda, "Gibbs phenomenon in structural control," J. Guidance, Contr., and Dynamics, vol. 14, no. 1, pp. 51-58, 1991.

[8] S. T. Venkataraman and S. Gulati, "Terminal slider control of nonlinear systems," in Proc. Int. Conf. Advanced Robotics, Pisa, Italy, June 1990

[9] E. R. Rang, "Isochrone families for second-order systems," IEEE Trans. Automat. Contr., vol. 8, pp. 64-65, Jan. 1963.

[10] S. V. Salehi and E. P. Ryan, "On optimal nonlinear feedback regulation of linear plants," IEEE Trans. Automat. Contr., vol. AC-27, pp. 1260-1264, Dec. 1982.

[11] V. T. Haimo, "Finite time controllers," SIAM J. Contr. Optim., vol. 24, pp. 760-770, July 1986

[12] S. P. Bhat and D. S. Bernstein, "Lyapunov analysis of finite-time differential equations," in Proc. Amer. Control Conf., Seattle, WA, June 1995, pp. 1831-1832.

[13] A. A. Andronov, A. A. Vitt, and S. E. Khaikin, Theory of Oscillators. Oxford, U.K.: Pergamon, 1966.

[14] S. P. Bhat and D. S. Bernstein, "Continuous, bounded, finite-time stabilization of the translational and rotational double integrators," in Proc. IEEE Conf. Control Applications, Dearborn, MI, Sept. 1996, pp. 185-190.

[15] P. Hartman, Ordinary Differential Equations, 2nd ed. Boston, MA: Birkhäuser, 1982.

[16] M. Kawski, "Stabilization of nonlinear systems in the plane," Syst. Contr. Lett., vol. 12, pp. 169-175, 1989.

[17] A. F. Filippov, "Differential equations with discontinuous righthand sides," Math. Its Appl., 1988.

\section{Comments on "An Algorithm for Real-Time Failure Detection in Kalman Filters"}

Thomas H. Kerr

\begin{abstract}
In the above-mentioned paper, ${ }^{1}$ we object to claims made of providing simpler implementation complexity or computational burden without an explicit mechanization for obtaining a solution ever actually being offered (and operations counts being tallied).
\end{abstract}

Index Terms - Constrained optimization, ellipsoid confidence region CR2 overlap test, failure detection, Kalman filter, RAIM.

We have provided frequent up-to-date surveys of the status of failure detection technology (elucidating the various emerging approaches) on seven previous occasions [1]-[5], [7], [8] in keeping abreast of this fast changing area. We have developed first hand, identified, specified, or recommended preferred implementations for particular application situations or scenarios, including that of detecting anomalous behavior of new navigation systems introduced on SSBN submarines [10]-[17] and for a multisensor navigation filter and failure detection, identification, and reconfiguration (FDIR) strategy in the advanced tactical fighter (ATF) involving multiple simultaneous navaids such as the joint tactical information and distribution system (JTIDS) and global positioning system (GPS) and the standard onboard Inertial navigation system (INS) [2], [3], [9].

Zolghadri claims [19, p. 1538, col. 2, par. 1] that the method in [15] leads to an unconstrained optimization problem that is complex to implement. We object on two points: first, [15] is a constrained optimization problem and, second, whose solution only requires finding the appropriate scalar Lagrange multiplier for a full solution in just five evaluation steps [15, p. 523, Fig. 7]. We specify the successive approximations iteration equation for it and prove convergence as a contraction mapping (with geometric convergence rate) and implement it in real-time in [10]-[12], as reported in sanitized (unclassified) form in [14]-[17].

The assertion in $[19$, p. 1539 , col. 1, step ii] is that Zolghadri has a technique of lower computational burden than the earlier scalar iteration equation of $[15$, p. 519 , eq. 34], shown in (1) at the top of the next page, for fixed $w(k) \triangleq\left[\hat{x}_{1}(k)-\hat{x}_{2}(k)\right]$ and fixed time-step $k$ in the above, starting with an initial guess of 0.75 for convenience to be iterated on $n$ to convergence for obtaining the scalar Lagrange multiplier $\lambda^{*}$, but Zolghadri does not give a method for specifying or solving for his corresponding optimal $\lambda_{0}$ to substantiate his claim of offering a reduced computational burden (even though Zolghadri asserts that the "dichotomy method allows one to approach rapidly the negative root of $F(\lambda)$ " in [19, p. 1539, col. 1, pt. ii], there is not enough detail presented there or in [18] to allow a confirmation). Initial indications are, even without Zolghadri explicitly specifying an iteration equation (since his corresponding equations ([19, p. 1539, step ii]) are

$$
F(\lambda)=\Omega^{T}(\lambda) P_{2}^{-1}(k) \Omega(\lambda)-\alpha
$$

Manuscript received October 25, 1996.

The author is with TeK Associates, Lexington, MA 02173-0003 USA (email: tkerr@tiac.net).

Publisher Item Identifier S 0018-9286(98)03609-5.

${ }^{1}$ A. Zolghadri, IEEE Trans. Automat. Contr., vol. 41, pp. 1537-1539, Oct. 1996. 